\title{
Contenção do paciente agitado no Departamento de Emergência
}

\author{
Rodrigo Fabian Castillo Schmidt, MD ${ }^{1,2^{*}}$ \\ ${ }^{1}$ Hospital Israelita Albert Einstein, São Paulo, Brasil. \\ 2 Hospital das Clínicas da Faculdade de Medicina da Universidade de São Paulo, São Paulo, Brasil. \\ *Autor correspondente. Endereço de e-mail: rodrigo.schmidt@hc.fm.usp.br
}

O paciente agitado, é uma realidade cotidiana no Departamento de Emergência, e assim como uma apresentação corriqueira, deveríamos estar amplamente treinados e qualificados para abordar estes pacientes, com a destreza, agilidade e humanidade que tratamos qualquer outro, pois são pessoas que chegam a nossas mãos precisando ajuda, seja para garantir sua segurança até um surto psicótico terminar, ou até que a causa seja identificada e revertida.

Infelizmente, esta não é a conduta habitual nos departamentos de emergência. Em geral, observamos um conjunto de incertezas, ataduras e tom de voz ameaçador.

Isto acontece pelo desconhecimento de alternativas químicas eficazes para contenção ficando apenas a alternativa mecânica de amarrar o paciente até o haloperidol atingir lenta e ineficazmente a sedação, sem esquecer de passar esses longos minutos torcendo, para que a prometazina nos ajude ativando seu efeito colateral de depressão do SNC.

Nesta revisão iremos apresentar essas alternativas e como deverão ser escolhidas. Vamos nos adentrar no excitante mundo das drogas sedativas, visando uma contenção química, rápida, eficaz e humana. Você, sua equipe, e especialmente seu paciente, serão os beneficiados.

A tabela 1 apresenta as causas mais comuns de agitação encontradas no departamento de emergência. Não cabe no escopo desta revisão entrar em detalhes e discutir essas causas.

Tabela 1. Causas perigosas de agitação psicomotora.

\begin{tabular}{l}
\hline \hline \multicolumn{1}{c}{ Etiologia } \\
\hline Sepse \\
Trauma craniano \\
AVC isquêmico / hemorrágico \\
Massa intracraniana
\end{tabular}

Infecção de sistema nervoso central

Crises convulsivas

Distúrbios hidroeletrolíticos

Hipoglicemia

Hiperglicemia

Hipóxia

Hipercarbia

Insuficiência renal

Encefalopatia hepática

Deficiência nutricional (Vitamina B1, B3 ou B12, por exemplo)

Intoxicação (anticolinérgico, estimulante, alcool, metanol, monóxido de carbono)

Psicose associada a corticóide

Síndrome serotoninérgica

Síndrome neuroléptica maligna

Hipertermia / Hipotermia

Choque

Psicose

Esquizofrenia

Delírio paranóide

Distúrbio de personalidade

\section{Classificação de agitação}

Antes que nada, precisamos individualizar nossa abordagem. Identificar com quem estamos lidando, para definir nossas estratégias. Para isto iremos classificar nosso paciente em três grupos:

Grau 1 - Ansioso e agitado, porém, colaborativo.

É aquele paciente que está ciente do seu estado e demanda atenção. Por essa característica, ele estará disposto a interagir, conversar e exigir coisas. Nos dando a oportunidade de conseguir acalmá-lo sem intervenção química parenteral, muito menos mecânica.

Uma vez atingida uma linha de comunicação, ter facilitado um alimento ou bebida, demonstrando nosso interesse no seu bem-estar, podemos explicar o benefício de um agente que promova ansiolise, para ele conseguir relaxar e descansar. 
Grau 2 - Disruptivo e destrutivo, porém sem conduta ameaçadora para consigo mesmo ou a equipe.

É aquele paciente que joga objetos no chão, rasga a roupa, grita, e só faz contato para ofender, e manifestar que não quer nada.

Este perfil de paciente é o primeiro que precisa abordagem química parenteral, pois a deficiência na comunicação tornara a situação, longa, exaustiva e frustrante. Inclusive podendo dar chance a um escalonamento no grau de agitação, tornando o paciente agressivo.

Grau 3 - Agressivo, incapaz de se comunicar. Representa risco iminente de auto e heteroagressão.

É aquele paciente que pode colocar em risco sua própria integridade, assim como a da equipe assistencial. Os pacientes grau 3, precisam de contenção rápida e eficaz.

Cabe lembrar, que para todos os pacientes agitados, sejam Grau 1, 2 ou 3, a inversão da escalada da violência ou a contenção do seu estado comportamental configura só o primeiro passo de uma abordagem completa e resolutiva. Integra também esse primeiro passo identificar e tratar causas de dito estado. Devemos entender que a agitação psicomotora, pode ser uma manifestação cardinal de um quadro fisiopatológico grave, indo muito além do típico paciente psiquiátrico em surto, ao que relacionamos quase sempre a este evento.

\section{Abordagem inicial de acordo com a classificação}

Uma vez classificado o grau de agitação do nosso paciente, é o momento de definir nossa abordagem, empregando ferramentas que permitam prosseguir nosso atendimento de maneira rápida e assertiva.

Grau 1 - Velocidade - / Segurança +++

- Contato verbal, acolhedor, expressando nosso interesse em ajudar.

- Ser flexível a demanda simples, como alimento e bebida adequada para o consumo dentro do centro assistencial.

- Temporizar demandas que não podem ser acolhidas imediatamente.

- Demarcar limites, estabelecendo com voz clara e firme, que não será admitida qualquer conduta ofensiva ou disruptiva.

- Caso a disrupção esteja interferindo no restante do funcionamento do departamento de emergência, considerar mudar o paciente de lugar, afastando-o dos demais pacientes que aguardam.

Finalmente, podemos oferecer a possibilidade de receber agente $\mathrm{VO}$, para relaxar e descansar.

Agentes de escolha:

- Lorazepam 1 - 2 mg

-Alprazolam 0,5 - $1 \mathrm{mg}$

Outros:

- Risperidona $2 \mathrm{mg}$

- Olanzapina 5 - $10 \mathrm{mg}$

Grau 2 - Velocidade + / Segurança ++

- Sempre abordar inicialmente com as medidas de inversão de escalada de violência citadas para o Grau 1.

- Mas esta tentativa será limitada ao tempo que a equipe precise para se preparar para a contenção física e administração do agente químico.

Agente de escolha:

- Droperidol 5 - $10 \mathrm{mg}$ (IM)

Outros:

- Haloperidol $10 \mathrm{mg}$ + Midazolam 2 - 2,5 mg (IM)

- Midazolam 5 - $10 \mathrm{mg}$ (IM)

Grau 3 - Velocidade +++ / Segurança +

- Pelas características agressivas de estes pacientes, a intervenção precisa ser rápida e eficaz. Visando controle total da situação e do indivíduo, garantindo segurança tanto nas manobras físicas como na vigilância de efeitos colaterais que possam acontecer como complicações na intervenção empregada.

- Em geral será necessário a contenção manual descrita abaixo.

$\mathrm{O}$ agente que possui o perfil mais adequado para nosso objetivo, é a quetamina.

Agente de escolha:

- Quetamina 4 -5 mg/kg (IM)

\section{Funcionamento das medicações}

\section{Lorazepam}

Benzodiazepínico de primeira escolha, pelas suas características farmacológicas. Mas ausente na maioria de centros assistenciais.

\section{Alprazolam}

Benzodiazepínico com bom início de ação, excelente biodisponibilidade VO, meia vida relativamente curta visando alta precoce. Características muito semelhantes ao lorazepam. 
Tabela 2. Descrição do funcionamento das medicações.

\begin{tabular}{lllll}
\hline \hline & Dose & Início de ação & Pico de ação & Meia-vida* \\
\hline \hline Lorazepam (VO) & $0,5-2 \mathrm{mg}$ & $20-30 \mathrm{~min}$ & $2 \mathrm{~h}$ & $12 \mathrm{~h}$ \\
Alprazolam (VO) & $0,5-1 \mathrm{mg}$ & $30 \mathrm{~min}$ & $1-2 \mathrm{~h}$ & $12 \mathrm{~h}$ \\
Risperidona (VO) & $1-2 \mathrm{mg}$ & $60 \mathrm{~min}$ & $1 \mathrm{~h}$ & $20 \mathrm{~h}$ \\
Olanzapina (VO) & $5-10 \mathrm{mg}$ & $120 \mathrm{~min}$ & $6 \mathrm{~h}$ & $30 \mathrm{~h}$ \\
Quetiapina (VO) & $25 \mathrm{mg}$ & $60 \mathrm{~min}$ & $1.5 \mathrm{~h}$ & $6 \mathrm{~h}$ \\
Droperidol (IM) & $5-10 \mathrm{mg}$ & $3-10 \mathrm{~min}$ & $0.5 \mathrm{~h}$ & $2 \mathrm{~h}$ \\
Droperidol (EV) & $2.5-10 \mathrm{mg}$ & $2-5 \mathrm{~min}$ & $5 \mathrm{~min}$ & $2 \mathrm{~h}$ \\
Haloperidol (VO) & $2-10 \mathrm{mg}$ & $2 \mathrm{~h}$ & $2-6 \mathrm{~h}$ & $14-37 \mathrm{~h}$ \\
Haloperidol (IM) & $2-10 \mathrm{mg}$ & $15 \mathrm{~min}$ & $20 \mathrm{~min}$ & $20 \mathrm{~h}$ \\
Haloperidol (EV) & $2-10 \mathrm{mg}$ & $3 \mathrm{~min}$ & $30 \mathrm{~min}$ & $14-26 \mathrm{~h}$ \\
Midazolam (IM) & $2,5-5 \mathrm{mg}$ & $15 \mathrm{~min}$ & $15-30 \mathrm{~min}$ & $3-5 \mathrm{~h}$ \\
Midazolam (EV) & $2,5-5 \mathrm{mg}$ & 1 a $5 \mathrm{~min}$ & $3-5 \mathrm{~min}$ & $2-6 \mathrm{~h}$ \\
Quetamina (IM) & $4-6 \mathrm{mg} / \mathrm{kg}$ & $5 \mathrm{~min}$ & $5-30 \mathrm{~min}$ & $2,5 \mathrm{~h}$ \\
Quetamina (EV) & $1-2 \mathrm{mg} / \mathrm{kg}$ & $30 \mathrm{segundos}$ & $30 \mathrm{segundos}$ & $2,5 \mathrm{~h}$ \\
\hline
\end{tabular}

*Meia-vida considerando função renal e hepáticas normais.

\section{Droperidol}

Neuroléptico do grupo das butirofenonas, com possibilidade de administração tanto IM com EV. Possui um início muito adequado e meia vida curta no contexto de manejo do paciente agitado.

\section{Haloperidol}

Neuroléptico que também pertence ao grupo das butirofenonas, porém com perfil muito menos interessante que o droperidol. A rigor, sua administração EV pode favorecer o tempo de início, mas cabe lembrar que essa via de administração mesmo estando relatada na literatura, se configura como off-label. Em termos gerais, ele oferece um início de ação lenta, entre 15 - 30 min e uma meia vida prolongada aproximadamente $18 \mathrm{~h}$. Representa uma janela muito extensa para avaliar se a dose administrada foi adequada, e assim complicando a decisão de replicar as doses, com risco consequente de provocar acumulo plasmático da droga.

\section{Midazolam}

Benzodiazepinico, com excelente tempo para início de ação após administração EV, flexibilidade para uso IM. E uma meia vida curta, de 2-6h. Porém, a variação da dose entre sedação adequada e depressão do sistema respiratório é muito pequeno, tornando a necessidade de monitorização e eventual intervenção na via aérea, um risco ativo. Observação: é baseado nestes dados farmacológicos que optamos pela combinação de haloperidol na dose padrão/alta somado ao midazolam dose baixa. Visando a sinergia entre os agentes para atingir sedação alvo, num menor tempo de ação, e diminuindo o risco de depressão respiratória.

\section{Quetamina}

Antagonista dos receptores NMDA, que induz um estado de dissociação a estímulos externos, sem promover diminuição do perfil hemodinâmico, nem comprometer mecânica respiratória como um todo (drive ventilatório, proteção da via aérea e reflexos associados). Oferece ainda um curto início de ação via IM, 3-4 min na grande maioria de abordagens. No caso de administração endovenosa, não deve ser realizado em bolus pois existe risco de perda de drive ventilatório com necessidade de intervenção da via aérea.

\section{Descrição das condutas e manobras}

\section{Inversão da escalada de violência}


Esta técnica tem pontos chave para que seja bem sucedida. Todos se baseiam no fundamento de que $90 \%$ da carga emocional que transmitimos em uma mensagem, e $50 \%$ da informação total que podemos transmitir em uma língua falada, vem da linguagem corporal, e especialmente o tom de voz. Fixando esta informação na mente, podemos pontuar os seguintes itens como indispensáveis. situação"

Apoio: "Vamos nos ajudar para resolver a

Compreensão: "Vejo que toda essa situação está sendo difícil para você"

Validação: "Acho que eu também teria ficado chateado no seu lugar"

Resolução: "Vamos fazer o seguinte"

Designar uma única pessoa para realizar a abordagem. Realizá-la idealmente num ambiente calmo e intimista. Comunicar-se de forma honesta e real com o paciente olhando-o nos olhos. Evite dissimulação e ficar desviando o olhar, pois comunica a impressão de insinceridade. Sempre manter a distância de dois braços com o paciente evitando tocá-lo. Toques são interpretados como invasão do espaço pessoal e violência. Sempre contar com uma via de saída em direção contraria ao paciente.

\section{Contenção Manual (Hold and Release)}

É a técnica preferida de contenção física, pois é a mais humanizada. Esta técnica estabelece que a contenção do paciente será feito de uma forma segura por meio de abordagem corpo a corpo, sem materiais inanimados (correias, fitas, ataduras, etc) viabilizando a administração dos agentes químicos, que facilitarão a contenção definitiva do nosso paciente. Sendo simplesmente um coadjuvante ponte até que o efeito sedativo desejado seja atingido.

Esta manobra deve ser realizada por não menos do que cinco pessoas, não contando com o profissional que administrará a medicação. Cada uma delas devera centrar sua força em cada braço e antebraço, assim como nos joelhos e cabeça. A pessoa que fica a cargo da cabeça, deverá colocar máscara no paciente para oferta de $\mathrm{O} 2$, ao mesmo tempo de proteger a equipe de exposição a saliva ou mordidas.

\section{Contenção Mecânica (faixas, correias ou ataduras)}

Esta estratégia fica reservada para duas situações unicamente.

1) Casos extremos, onde o risco de auto ou heteroagressão seja iminente.
2) Janelas terapêuticas das medicações sedativas em que há risco de retirada acidental de dispositivos (tubo traqueal por exemplo), provocadas por diminuição de concentração plasmática dos agentes químicos empregados.

Estes eventos podem acarretar mais dano ao paciente. Caso precisemos desta medida, devemos preconizar seu uso seguindo as seguintes diretrizes:

- Usar materiais apropriados, que evitem lesão local por atrito.

- Os pontos de fixação deverão ser sempre ancorados na estrutura do leito, e não nas grades, ou peças móveis.

- A contenção deve ser aplicada em no mínimo 4 pontos no paciente. Sendo eles os 4 membros, evitando pulsos e tornozelos.

- Preconizamos a ancoragem de um membro superior por cima da cabeça e outro inferior ao nível da cintura.

- Enfaixamento do tórax, será reservado como último recurso. Caso seja necessário, a fixação deverá ser realizada na mesma altura que o tórax, e nunca em direção a cabeceira do leito. Confirmar que não há restrição ao movimento respiratório.

- Todas as fixações, deverão oferecer certa liberdade ao movimento, para assim garantir a ausência de comprometimento vascular da região.

- O decúbito da cama sempre deverá ser mantido levemente elevado para favorecer o fluxo fisiológico de secreções e visar conforto do paciente.

- Uma pessoa responsável deverá permanecer com o mesmo para oferecer comunicação e auxílio ante qualquer necessidade ou intercorrência.

\section{Prevenção de eventos adversos relacionados à contenção mecânica}

Como ponto principal devemos lembrar da segurança tanto de nossa equipe, quanto de nosso paciente.

Diante de alguns eventos recentes lamentáveis, relacionados a contenção física, temos a ela, como causa principal dos desfechos, que em alguns casos chegaram a desencadear o falecimento da vítima. Como por exemplo abordagens policiais.

Isto nos leva a reflexão. Podemos empregar a contenção física ou mecânica? A resposta é sim. 
Como abordado anteriormente, esta técnica poderá ser utilizada como ponte, até atingir controle químico do paciente. Em ocasiões extremas, poderá ser mantida por um período mais prolongado. Porém, devemos ter sempre presente que em qualquer uma destas situações, nunca deveremos incorrer nos seguintes erros.

1. Cobrir a boca, ou nariz com tecidos, objetos, ou mesmo a mão.

2. Exercer pressão ou restrição fora dos pontos previamente comentados, principalmente tórax, pescoço, costas e abdome.

3. Deixar de prestar atenção a eventuais pedidos coerentes do paciente, assim como monitorar alguma evidência de asfixia.

4. Prolongar a contenção por mais tempo do que o necessário.

5. Adiar a administração do agente sedativo.

Estes pontos se baseiam essencialmente em evitar as possíveis complicações que existem ao ter uma pessoa sob restrição mecânica. Como asfixia, hipertermia e rabdomiólise entre outras.

\section{Contexto legal da contenção mecânica}

Em abril de 2015, o Conselho Regional de Medicina do Estado de São Paulo (CREMESP) publicou o Parecer No. 175.956, após o Ministério Público do Estado de São Paulo questionar "se existem normas ou resoluções sobre regras e procedimentos acerca de contenções psiquiátrica", bem como "quais profissionais são habilitados para a realização de contenção".

Este parecer sustenta que a restrição é atribuição médica conforme descrito pelo Conselho Federal de Medicina (art.11 - Resolução 1.598, de 09 de agosto de 2000). É fundamentada no Código de Ética Médica, nas Resoluções e Pareceres do Conselho Federal de Medicina e nas Portarias do Ministério da Saúde que normatizam os Centros de Atenção Psicossocial (CAPS): Resolução CFM 2.057/2013, Resolução CFM 1.952/2010, Processo Consulta no 8.589/10 - CFM (01/11), Parecer no 1.317/01-CRMPR, Portaria 224/1992, Portaria MS/GM 336/2002, Portaria MS/GM 3088/2011 e Portaria MS/GM 121/2012. Aplica-se a situações em que a restrição é o meio mais adequado para prevenir dano imediato ou iminente ao próprio paciente ou a terceiro.

Por sua vez, o Conselho Regional de Enfermagem de São Paulo orienta que o profissional enfermeiro pode prescrever a contenção, se houver protocolo compartilhado autorizando da contenção. Aos técnicos e auxiliares de enfermagem cabe a execução da contenção sob supervisão do enfermeiro.

$\mathrm{Na}$ resolução do Conselho Federal de Enfermagem 427/2012, art. 1, reza que os profissionais de enfermagem, excetuando-se as situações de urgência e emergência, somente poderão empregar a contenção mecânica do paciente sob supervisão direta do enfermeiro e, preferencialmente, em conformidade com protocolos estabelecidos pelas instituições de saúde, públicas ou privadas, a que estejam vinculados.

Salienta-se que uns dos objetivos imediatos da contenção é a segurança tanto do paciente como da equipe assistencial. Faz-se relevante citar um parecer do Conselho Regional de Medicina do Estado do Paraná (Parecer 2.456/2014), onde ratifica, que profissionais de segurança, devidamente treinados, podem auxiliar a equipe médica e de enfermagem, na contenção.

\section{Conclusão}

Devemos relembrar que mesmo que a contenção do nosso paciente será um passo indispensável e prioritário. Ela só forma parte de uma estratégia integral do atendimento, para viabilizar uma estabilização efetiva, assim como uma assertiva investigação etiológica do evento.

\section{Referências}

1. Nazarian DJ, Broder JS, Thiessen MEW, et al. Clinical Policy: Critical Issues in the Diagnosis and Management of the Adult Psychiatric Patient in the Emergency Department. Ann Emerg Med. 2017;69(4):480-498.

2. Coburn, V. \& Mycyk, M. (2009). Physical and Chemical Restraints. Emergency Medicine Clinics of North America. 27, 655-667.

3. Rossi, J. Swan, M. \& Isaacs, E. (2010). The Violent or Agitation Patient. Emergency Medicine Clinics North America. 28, 235-256.

4. New A, Tucci VT, Rios J. A Modern-Day Fight Club? The Stabilization and Management of Acutely Agitated Patients in the Emergency Department. Psychiatr Clin North Am. 2017 Sep;40(3):397-410.

5. Nice.org.uk. (2018). Violence and aggression: short-term management in mental health, health and community settings | Guidance and guidelines 
I NICE. [online] Available at: https://www.nice.org.uk/guidance/ng10.

6. Wilson MP, Pepper D, Currier GW, Holloman GH Jr, Feifel D. The psychopharmacology of agitation: consensus statement of the American Association for emergency psychiatry project Beta psychopharmacology workgroup. West J Emerg Med. 2012 Feb;13(1):26-34.

7. Swift, RH, Harrigan, EP, Cappelleri, JC, et al, Validation of the behavioural activity rating scale (BARS): a novel measure of activity in agitated patients. Journal of Psychiatric Research, 2002. 36: p. 87-95.

8. Zeller SL, Citrome L. Managing Agitation Associated with Schizophrenia and Bipolar Disorder in the Emergency Setting. West J Emerg Med. 2016;17(2):165-72.

9. Richmond JS, Berlin JS, Fishkind AB, et al. Verbal De-escalation of the Agitated Patient: Consensus Statement of the American Association for Emergency Psychiatry Project BETA De-escalation Workgroup. West J Emerg Med. 2012 Feb;13(1):17-25.

10.Glezer A, Brendel RW. Beyond emergencies: the use of physical restraints in medical and psychiatric settings. Harv Rev Psychiatry. 2010 Nov-Dec;18(6):353-8.

11.Downey LV, Zun LS, Gonzales SJ, Frequency of alternative to restraints and seclusion and uses of agitation reduction techniques in the emergency department. Gen Hosp Psychiatry, 2007. 29(6): p. 470-4.

12. Martin B, Restraint use in acute and critical care settings: changing practice. AACN Clin Issues, 2002. 13(2): p. 294-306.

13.Centers for Medicare and Medicaid Services. State Operations Manual. Department of Health and Human Services; 2008. Available at: https://www.cms.gov/Regulations-and-

Guidance/Guidance/Transmittals/downloads/R37S OMA.pdf.

14.Zun LS. A prospective study of the complication rate of use of patient restraint in the emergency department. J Emerg Med. 2003 Feb. 24(2):11924.

15. Coburn VA, Mycyk MB. Physical and chemical restraints. Emerg Med Clin North Am. 2009 Nov. 27(4):655-67, ix.

16.Zun LS. A prospective study of the complication rate of use of patient restraint in the emergency department. J Emerg Med. 2003 Feb. 24(2):11924.
17.Annas GJ. The last resort-the use of physical restraints in medical emergencies. $\mathrm{N}$ Engl J Med. 1999 Oct 28. 341(18):1408-12.

18.Currier GW, Simpson GM, Risperidone liquid concentrate and oral lorazepam versus intramuscular haloperidol and intramuscular lorazepam for treatment of psychotic agitation. J Clin Psychiatry, 2001. 62(3): p. 153-7.

19. Currier GW, Chou JC, Feifel D, Acute treatment of psychotic agitation: a randomized comparison of oral treatment with risperidone and lorazepam versus intramuscular treatment with haloperidol and lorazepam. J Clin Psychiatry, 2004. 65(3): p. 386-94.

20.Nobay F, Simon BC, Levitt MA, Dresden GM. A prospective, double-blind, randomized trial of midazolam versus haloperidol versus lorazepam in the chemical restraint of violent and severely agitated patients. Acad Emerg Med. 2004;11(7):744-9.

21.Richards JR, Derlet RW, Duncan DR, Chemical restraint for the agitated patient in the emergency department: lorazepam versus droperidol. J Emerg Med, 1998. 16(4): p. 567-73.

22. Martel M, Sterzinger A, Miner J, et al, Management of acute undifferentiated agitation in the emergency department: a randomized double-blind trial of droperidol, ziprasidone, and midazolam. Acad Emerg Med, 2005. 12(12): p. 1167-72.

23. Isenberg DL, Jacobs D. Prehospital Agitation and Sedation Trial (PhAST): A Randomized Control Trial of Intramuscular Haloperidol versus Intramuscular Midazolam for the Sedation of the Agitated or Violent Patient in the Prehospital Environment. Prehosp Disaster Med. 2015 Oct;30(5):491-5.

24.Kroczak $S$ et al. Chemical Agents for the Sedation of Agitated Patient in the ED: A Systematic Review. Am J Emerg Med. 2016. (34); 2426-2431.

25. Gottlieb M, Long B, Koyfman A. Approach to the Agitated Emergency Department Patient. J Emerg Med. 2018;54(4):447-457.

26.Zun LS. Evidence-Based Review of Pharmacotherapy for Acute Agitation. Part 1: Onset of Efficacy. J Emerg Med. 2018;54(3):364374.

27.Gottlieb M, Schiebout J. What Is the Efficacy of Droperidol for the Management of Acute Psychosis-Induced Agitation?. Ann Emerg Med. 2018;71(1):141-143.

28. Battaglia J, Moss S, Rush J, et al. Haloperidol, lorazepam, or both for psychotic agitation? A 
multicenter, prospective, double-blind, emergency department study. Am J Emerg Med. 1997 Jul;15(4):335-40.

29. Bieniek SA, Ownby RL, Penalver A, Dominguez RA. A double-blind study of lorazepam versus the combination of haloperidol and lorazepam in managing agitation. 1998 Jan-Feb;18(1):57-62.

30. Hui D, Frisbee-Hume S, Wilson A, et al. Effect of Lorazepam With Haloperidol vs Haloperidol Alone on Agitated Delirium in Patients With Advanced Cancer Receiving Palliative Care: A Randomized Clinical Trial. 2017 Sep 19;318(11):1047-1056.

31.Le Cong M, Humble I. A Ketamine Protocol and Intubation Rates for Psychiatric Air Medical Retrieval. Air Med J. 2015 Nov-Dec;34(6):357-9.

32. Cole JB, Moore JC, Nystrom PC, et al. A prospective study of ketamine versus haloperidol for severe prehospital agitation. Clin Toxicol (Phila). 2016 Aug;54(7):556-62.

33. Riddell J, Tran A, Bengiamin R, Hendey GW, Armenian P. Ketamine as a first-line treatment for severely agitated emergency department patients. Am J Emerg Med. 2017 Jul;35(7):1000-1004.

34. Isbister GK, Calver LA, Downes MA, Page CB. Ketamine as Rescue Treatment for Difficult-toSedate Severe Acute Behavioral Disturbance in the Emergency Department. Ann Emerg Med. 2016;67(5):581-587.e1.

35.Vrana B. Use of Intranasal Ketamine for the Severely Agitated or Violent ED Patient. J Emerg Nurs. 2016;42(3):198-9.

36. Lahti AC, Koffel B, LaPorte D, Tamminga CA. Subanesthetic doses of ketamine stimulate psychosis in schizophrenia. 1995 Aug;13(1):9-19.

37.Peisah C, Chan DK, McKay R, Kurrle SE, Reutens $S G$. Practical guidelines for the acute emergency sedation of the severely agitated older patient. Intern Med J. 2011 Sep;41(9):651-7.

38. Aftab A, Shah AA. Behavioral Emergencies: Special Considerations in the Geriatric Psychiatric Patient. Psychiatr Clin North Am. 2017 Sep;40(3):449-462.

39. Kruse WH. Problems and pitfalls in the use of benzodiazepines in the elderly. Drug Saf. 1990 Sep-Oct;5(5):328-44.

40.Bogunovic OJ, Greenfield SF. Practical geriatrics: Use of benzodiazepines among elderly patients. Psychiatr Serv. 2004 Mar;55(3):233-5. 